\title{
Nota Farmacológica: ¿Es adecuado prescribir anticonceptivos orales (ACO) en mujeres mayores de 35 años?
}

Adaptado de: Prescribing Oral Contraceptives for Women Older Than 35 Years of Age. Christine Seibert, MD Ellen Barbouche. Ann Intern Med 2003; 138:54-64.

\section{Resumen para llevar al consultorio}

En la presente nota se ejemplifica con un caso clínico habitual, los factores a tener en cuenta al prescribir anticonceptivos orales (ACO) a mujeres de más de 35 años.

Los ACO son seguros y pueden ser prescriptos en mujeres mayores de 35 años, grupo en los que son subutilizados.

Hay que tener en cuenta que pueden aumentar el riesgo de ACV, IAM, y eventos tromboembólicos (aunque muy bajo a nivel individual, no es despreciable a nivel poblacional). El riesgo aumenta principalmente en fumadoras e hipertensas no controladas así como en las que tienen antecedentes tromboembólicos o múltiples factores de riesgo.

Se señalan las contraindicaciones de la OMS par los ACO.

Por otro lado beneficios adicionales a la prevención del embarazo son la reducción del cáncer de ovario, de endometrio, posiblemente de colon, la mejora del acné e hirsutismo, y de los síntomas perimenopáusicos y de la densidad ósea.

Una mujer de 42 años viene a verlo por que quiere reiniciar su actividad sexual luego de cinco años, cuando se divorció de su marido. En el pasado ha tolerado bien los anticonceptivos orales (ACO) y le plantea reiniciarlos.

\section{¿Cuál es el potencial riesgo asociado con los ACO durante Ios últimos años de la vida reproductiva?}

Muchas mujeres y médicos creen que los ACO se asocian a riesgos sustanciales después de los 35 años. En EE.UU. sólo el 4\% los toman.Parte de la preocupación responde a estudios tempranos en donde se demostraban aumento de la incidencia de efectos adversos, algunos graves, como el tromboembolismo venoso. Su incidencia es mayor con dosis mayores a 50 ug de etinilestradiol (EE) y disminuye significativamente con dosis de 35 ug. Se supuso que con los nuevos preparados con dosis menores la incidencia sería menor, pero nuevos estudios comparando dosis de 20 ug vs $30-35$ ug no lo han podido demostrar.La edad no es determinante de dicho aumento. Globalmente, el riesgo de tromboembolismo venoso es bajo, se calcula en 1 caso extra por cada 10.000 usadoras de anticonceptivos por año.

Los ACO tienen diferentes progestágenos. Los más nuevos, también llamados de tercera generación son gestodeno, desogestrel y norgestimato.Si bien se han asociado a beneficios en el perfil lipídico y disminución de la androgenicidad (útil en acné e hirsutismo), dos grandes estudios caso-control ${ }^{\star}$ sugirieron que las pacientes que tomaban ACO conteniendo gestodeno y desogestrel tenían un $50 \%$ mayor de incidencia de trombosis venosa profunda y tromboembolismo pulmonar que las que consumían ACO con levonorgestrel. La investigación posterior reveló errores sistemáticos que desecharon las conclusiones previas.

Si bien el infarto agudo de miocardio (IAM) es raro en mujeres premenopáusicas, varios trabajos caso-control que ealizaron análisis multivariable* para ajustar por factores de riesgo coronario (FRC), han demostrado un aumento de riesgo, del orden de 3 a 5 veces en mujeres mayores a 35 años. Este aumento es mayor cuando se asocian otros FRC como tabaquismo (existe un efecto dosis dependiente, y es significativo con más de 10 cigarrillos por día), hipertensión, diabetes y dislipidemia.No se han encontrado diferencias estadísticamente significativas cuando se compararon progestágenos de tercera generación con los de primera y segunda.

Accidente cerebro vascular isquémico: estudios caso-control en varios países han demostrado resultados que varían entre el no aumento de riesgo y el aumento a 2 veces de del riesgo relativo de ACV. En el brazo europeo del estudio de la Organización Mundial de la Salud (OMS), no se demostró un aumento de ACV incluso en mujeres mayores de 35 años con dosis de EE de menos de 50 ug por día si tenían la presión controlada antes del inicio de los ACO; pero el riesgo relativo aumentaba a 7 y 10 si fumaban más de 10 cigarrillos por día o tenían una historia de HTA respectivamente. Un meta-análisis* reciente que analizó estudios con dosis de EE menores a 50 ug y ajustaron el riesgo de tabaquismo e HTA reveló una duplicación de riesgo de ACV en mujeres tomadoras de ACO.

En mujeres menores de 45 años con migrañas, y más aún en aquellas con aura, el riesgo de ACV isquémico está aumentado. La fuerza de tareas de la Sociedad Internacional de Cefaleas publica que hay un sinergismo entre la migraña y los ACO para el ACV. La OMS publica que el uso de ACO en mujeres menores a 35 años con migraña sin síntomas neurológicos focales es seguro, la recomendación general es evitarlos en mujeres de 35 años o mayores. En mujeres con síntomas neurológicos focales por migraña el uso de ACO está contraindicado, independientemente de la edad de la paciente.

Cáncer de mama: la evidencia es controvertida. Estudios casocontrol no han demostrado un aumento de riesgo.Un reanálisis de 54 estudios ha mostrado un aumento de $24 \%$ del riesgo en mujeres de cualquier edad que toman ACO en la actualidad que disminuía luego de la suspensión, sin aumento en las mayores de 35 años.De interés era que estos tumores eran más localizados que en la mujeres que nunca habían tomado ACO. Aunque algunos reportes recientes sugieren aumento del riesgo en mujeres con historia familiar positiva, la recomendación general es no contraindicarlos.

Litiasis vesicular: la litiasis sintomática aumenta con la paridad, la edad y el uso de ACO.Estudios de cohortes* retrospectivas han mostrado que las mujeres que toman ACO tienen un $10 \%$ de aumento del riesgo, independiente de la edad y que la paridad es un factor más decisivo que el uso de ACO.

Un grupo de tareas de la OMS desarrolló criterios de elegibilidad basados en la evidencia para el uso de ACO con 35 ug de estrógenos o menos. Para evitar riesgos de complicaciones serias los médicos debemos revisar las contraindicaciones absolutas de la OMS y aplicarlas en cada paciente a quien vamos a prescribir estos preparados. Se puede resumir como todos aquellos factores, condiciones o enfermedades que aumentan significativamente el riesgo de trombosis, IAM o ACV (ver Tabla 1) 


\section{Tabla 1: contraindicaciones absolutas de la OMS para uso de ACO}

- Embarazo

- 6 semanas postparto y amamantamiento

- = ó > 35 años y tabaquismo de mas de 15 cigarrillos por día

- TA: $160 / 99$ o mayor

- HTA con enfermedad vascular

- DBT con retino, nefro, neuropatías o enfermedad vascular

- Historia de trombosis venosa profunda o tromboembolismo de pulmón

- Cirugía mayor con prolongada inmovilización

- Historia de IAM o ACV

- Enfermedad valvular complicada ( con fibrilación auricular, hipertensión pulmonar, endocarditis bacteriana)

- Cefaleas severas con síntomas neurológicos focales

- Cáncer de mama actual

- Hepatitis viral activa, cirrosis severa, tumores hepáticos benignos o malignos

\section{¿Cuales son los beneficios potenciales?}

En los EE.UU., casi la mitad de las mujeres de más de 35 años tienen embarazos no deseados.Los ACO son uno de los métodos anticonceptivos más efectivos. Además del conocido beneficio anticoncepcional, reducen el riesgo de cáncer de ovario y de endometrio.Un meta-análisis* reciente de 17 estudios caso-control ${ }^{*}$ y tres estudios de cohortes ${ }^{*}$ confirmaron una reducción de cáncer de ovario de 10 a $12 \%$ el primer año y $50 \%$ luego de cinco años de uso. Otro meta-análisis* de 10 estudios caso-control* y un estudio de cohorte* mostró una reducción de riesgo de cáncer endometral de 56,67 y $72 \%$ luego de 4,8 y 12 años de uso de ACO respectivamente. La protección se mantiene muchos años luego de la suspensión.

Cáncer de colon: tres de cuatro estudios de cohortes ${ }^{*}$ y cinco de 11 estudios caso-control ${ }^{*}$ han mostrado reducción del riesgo con dosis mayores a 20 ug de estrógenos diarios. No es claro qué sucede con menores dosis. Aún no hay suficiente evidencia para recomendar ACO para este propósito.

Perimenopausia: las fluctuaciones de la secreción hormonal durante este período pueden preceder hasta en ocho años la menopausia y se caracterizan por los conocidos síntomas de calores, irritabilidad, insomnio, irregularidades menstruales, sequedad vaginal. Los ACO alivian todos estos síntomas, excepto los efectos sobre la disforia que son variables. La terapia de reemplazo hormonal estándar tiene cuatro a diez veces menor potencia que los ACO, no previene el embarazo, y hoy en día su uso se desaconseja para indicaciones preventivas poblacionales (no así para uso sintomático a mediano plazo).

Acné: dos estudios aleatorizados controlados demostraron mejoría del acné con el uso de norgestimato trifásico con 35 ug de EE durante 6 meses. Esta es la primera formulación aprobada por a FDA para el tratamiento del acné moderado. Todas las formulaciones de ACO, especialmente aquellas con progestágenos de tercera generación, pueden mejorar el acné a través del aumento de la globulina ligadora de hormonas sexuales inducida por estrógenos y una disminución de la testosterona libre sérica. En un $5 \%$ de los casos el acné puede empeorar en las usadoras de ACO. La misma disminución androgénica es la causa de la mejoría del hirsutismo, efecto que sumado al control del ritmo menstrual produce mejoría sintomática en el síndrome de ovario poliquístico. Dos revisiones recientes han señalado un efecto beneficioso sobre la densidad mineral ósea pero, aunque tres estudios han evaluado este beneficio en relación con la tasa de fracturas, los resultados son conflictivos y no se pueden sacar conclusiones.

Ud discute con su paciente riesgos y beneficios de Ios ACO. Sus menstruaciones son regulares, cada 30 días, con flujo moderado y leve síndrome premenstrual. No tiene historia personal ni familiar de embolias, trombosis, migrañas, ni cáncer ginecológico. Sólo toma ibuprofeno para la dismenorrea. Fuma 3 o 4 cigarrillos por día los fines de semana. Toma 1 o 2 copas de alcohol por semana y no hace actividad física. Su madre tiene hipercolesterolemia y ha tenido enfermedad coronaria a los 60 años.

\section{¿Qué examen físico y laboratorio debe hacerse a una mu- jer mayor a 35 años a quien se le va a prescribir ACO?}

Varias organizaciones profesionales incluyendo a la OMS y al Colegio Nor teamericano de Obstetricia y Ginecología determinan que el examen pelviano no es necesario antes del comienzo de los ACO ya que la historia clínica y la toma de tensión arterial son suficientes para evaluar las contraindicaciones. El examen de mamas y la mamografía están discutidas como rastreo antes de los 50 años de edad y no son necesarios per se en mujeres que van a recibir ACO.La evaluación de lípidos en ayunas está indicada a mujeres menores de 45 años que tienen otros FRC o historia familiar o personal de dislipidemia. Cualquiera de estas medidas pueden hacerse en la evaluación previa o en las visitas de seguimiento, no son necesariamente obligatorias antes de indicar ACO.

En el examen, la paciente tiene $138 / 88$ de TA y 88 latidos por minuto de FC. Sus mamas y examen pelviano son normales, al igual que su PAP. CT: 216; LDL: 145; HDL: 41; TG: 150 mg\%.

\section{¿Su presión levemente aumentada contraindica el uso de ACO?}

Los ACO pueden aumentar la presión sistodiastólica entre 4 y 9 $\mathrm{mm}$ de $\mathrm{Hg}$ desde el basal.Los valores vuelven a los niveles de pretratamiento luego de 3 a 6 meses de la discontinuación. Por el aumento marginal del riesgo de IAM y ACV isquémico en las mujeres hipertensas los médicos debemos ser cautelosos en la indicación de ACO aún en mujeres con leves elevaciones de la TA. No está contraindicado su uso pero requiere un monitoreo más cuidadoso de la TA en las visitas subsiguientes y se le debe aconsejar fuertemente que deje de fumar.

\section{¿Su dislipidemia leve contraindica el uso de ACO?}

Los ACO son en general seguros en esta situación si los lípidos se monitorean luego del inicio del uso.El Colegio Norteamericano de Obstetricia y Ginecología sugiere posponer la indicación hasta mayor control cuando la LDL sea = ó > 160, el HDL < 35 ó los triglicéridos $>250 \mathrm{mg} \%$.El efecto de los estrógenos sobre los lípidos depende de la dosis de estrógeno relativa a progestágenos, 
en adición a la androgenicidad del componente progestacional. Los estrógenos tienen un efecto beneficioso disminuyendo la LDL y aumentando HDL, aunque también aumentan los triglicéridos. Los progestágenos tienen un efecto opuesto, aumentando LDL y disminuyendo HDL.Los de tercera generación y más aun norgestimato, sin embargo, tienen más beneficio ya que aumentan HDL sin modificar LDL o manteniéndola estable, pero también aumentan los triglicéridos. Sin embargo, no hay datos que demuestren una disminución del riesgo cardiovascular.

\section{¿Su tabaquismo contraindica el uso de ACO?}

Un $25 \%$ de las mujeres de 35 a 45 años de edad fuman en EE.UU. la mayoría más de 15 cigarrillos por día, lo cual es desafortunado por el conocido aumento del riesgo cardiovascular. El riesgo absoluto estimado de muerte por enf ermedad coronaria en mujeres que consumen ACO y fuman es de 3,3 por 100.000 entre 15 y 34 años de edad comparado a 29,4 por 100.000 entre los 35 y 44 años de edad.Los paneles de consenso recomiendan precaución en prescribir ACO a mujeres mayores a 35 años que fuman. Se acepta generalmente que se contraindique en este grupo que fumen más que 15 cigarrillos por día.En fumadoras de menos cantidad se puede considerar pero no hay demasiados datos de la literatura que comparen estas dos categorías de tabaquistas. Lo más importante es que todas deben recibir un fuerte consejo del médico para dejar de fumar.

Ud discute con su paciente la necesidad de monitoreo de presión arterial y lípidos. Le aconseja fuertemente dejar de fumar, adoptar una dieta pobre en grasas y comenzar a hacer actividad física. Ella es receptiva y elige una fecha para dejar de fumar. Ud y su paciente acuerdan en que los ACO son una razonable opción para ella.

\section{¿Qué ACO debe ser prescripto?}

Una píldora con la dosis más baja efectiva de estrógenos. Como los ACO con 20 ug de EE por comp.no exhiben efectos adversos sobre la coagulación in vitro, este tipo de pastillas deben elegirse para pacientes que fuman. Sin embargo no hay estudios que demuestren menor riesgo cardiovascular en tomadoras de $20 \mathrm{ug}$ de EE por día. En contraposición sí hay evidencia que las píldoras de 35 ug de EE son mejores en disminuir el riesgo cardiovascular comparadas con las de 50 ug de estrógenos o más. No hay evidencia que el progestágeno afecte el riesgo cardiovascular, pero si modifica el riesgo de tromboembolismo venoso.

Ud discute algunos de los efectos adversos de los ACO con la paciente que se preocupa especialmente por el posible aumento de peso.

\section{¿Cuáles son los efectos adversos de los ACO?}

Un $25 \%$ de las mujeres experimentan efectos adversos menores mientras consumen ACO, la mayoría durante los tres primeros meses.Como son causa de adherencia limitada o interrupción del tratamiento, es importante educar al paciente acerca de los efectos adversos esperados y formas de prevenirlos. La razón más común de discontinuación es el sangrado menstrual anormal, segui- do de nauseas, ganancia de peso, cambios del ánimo, tensión mamaria, y cefalea. Los ACO de menor dosis de estrógenos van a disminuir la posibilidad de estos efectos adversos pero aumentará la posibilidad de sangrado.Nauseas y cefalea se asocian con dosis altas de EE. Las primeras desaparecen usualmente luego del segundo o tercer ciclo y pueden disminuir tomando la píldora con la cena o antes de dormir. La cefalea se relaciona con vasoespasmo o retención de fluidos. Cuando es persistente o severa merece una especial evaluación por que puede deberse a una complicación. La cefalea por retención de fluidos se acompaña de edemas, borborigmos y ganancia de peso, o síntomas mamarios. La ganancia de peso, cambios del ánimo y disminución de la libido son preocupaciones mayores para las pacientes. La primera, teóricamente, se puede producir por incremento de apetito por la progesterona o retención hidrosalina cíclica por el EE. Los cambios en el ánimo y la libido disminuida se relacionan con el componente progestacional y se desarrollan en menos del $20 \%$ de las mujeres.

\section{¿Cuán frecuentemente debe ser seguida?}

La mayoría recomienda seguimiento anual. Sin embargo en nuestra paciente cuya presión está levemente aumentada se aconseja control de presión en dos o tres meses.Si la presión se mantiene siempre por encima de 140/90 o llega a 160/100 los ACO deben ser interrumpidos hasta su adecuado control o indefinidamente. Sería aconsejable chequear los lípidos en nuestra paciente por sus antecedentes cada tres-seis meses y luego anualmente si se mantienen estables.EI PAP no debe hacerse más frecuentemente que en mujeres que no toman ACO. A todas las mujeres se les deberá aconsejar que consulten si tienen "ACHES", esto es dolor abdominal, dolor torácico, cefaleas, problemas oculares, dolor severo de piernas (regla mnemotécnica del inglés: abdominal o chest pain, headaches, eye problems, severe calf or thigh pain). Estos síntomas pueden indicar trombosis mesentérica, litiasis vesicular sintomática, tromboembolismo de pulmón, IAM, empeoramiento de migraña o ACV, embolia ocular, trombosis venosa profunda o tromboflebitis.

Dos meses después la paciente llama por sangrado tipo goteo una semana antes de su menstruación normal. Ella cuenta que dejó de fumar luego de la última visita.

\section{¿Como se maneja el sangrado?}

¿Su evaluación diagnóstica es diferente en mujeres de más de 35 años?

Su frecuencia es de 10 a $30 \%$ dentro del primer mes de consumo. Por su habitual resolución dentro de los primeros pocos ciclos se recomienda alentar a la mujer a continuar su uso.No hay evidencia de que algunos preparados sean superiores a otros pero en general aumenta la posibilidad con los de menores dosis de EE. Tomar la píldora todos los días a la misma hora ayuda a evitar el sangrado anor mal. El cigarrillo aumenta este efecto adverso por su efecto antiestrogénico que provoca disminución de su efectividad. Otras causas asociadas son Clamidia Trachomatis, interacciones medicamentosas, absorción disminuida, embarazo ectópico, endometrosis, endometritis, miomas, pólipos, cáncer endometral, displasia cervical. Si la paciente continúa con sangrado luego de tres meses sería aconsejable realizar un PAP, evaluar posible embarazo, y realizar un cultivo de flujo. También se puede cambiar de píldora, indicar antiinflamatorios no esteroides o un curso extra de estrógenos. 
El sangrado desaparece luego del tercer mes y ella no tiene más efectos adversos. Su presión es de $132 / 84$ a los 3 meses. Al año sus lípidos mejoraron algo. Sigue con ACO por varios años. A los 50 ella desea saber si ha entrado en menopausia.

\section{¿Cómo se diagnostica la menopausia en una mujer que toma ACO?}

Aunque los ACO son seguros en la quinta década de la vida, no previenen la menopausia.La concepción ha sido descripta aún a los 56 años, por lo cual es imprescindible saber que la mujer ya no requiere anticoncepción. Después de los 35 años los niveles de FSH y LH aumentan y los de estradiol disminuyen. La FSH mayor a $30 \mathrm{IU} / \mathrm{L}$ en una mujer que no menstrúa se considera indicativa de menopausia. A pesar que el diagnóstico habitual de menopausia en estas mujeres se hace con FSH elevadas a los 714 días post-suspensión del ACO, en algunos casos la FSH no aumenta. Más aún, algunas mujeres tienen FSH alta y amenorrea seguidas de ciclos normales. La terapia de reemplazo hormonal $(\mathrm{TRH})$ debe ser evaluada con la paciente. En mujeres que no requieren anticoncepción la $\mathrm{TRH}$ puede ser instaurada luego de los 50 años.Si la mujer requiere anticoncepción puede continuarla hasta los 55 años, ya que luego de esa edad la concepción es extremadamente improbable

\section{Conclusión}

Los ACO son seguros y pueden ser prescriptos en mujeres mayores de 35 años. Numerosos beneficios no relacionados a la anticoncepción pueden ser de interés en este grupo.Aunque más estudios en mujeres perimenopáusicas son necesarios, suficiente evidencia avala su utilización. Los médicos debemos educar acerca de los beneficios y los mitos para permitir que más mujeres elijan esta opción.

\section{Comentario}

Esta es una excelente revisión que cuestiona el bajo porcentaje de prescripción de anticonceptivos en mujeres de 35 años y más. Sin duda los ACO son de primera línea como anticonceptivos, tienen otros beneficios y no están contraindicados en mujeres de esta edad, si bien debemos recordar que es muy importante detectar a las fumadoras, hipertensas y mujeres con alto riesgo de enfermedad cardiovascular y tromboembólica para modificar su riesgo. Como en el caso citado, la evaluación de la medicación puede ser de utilidad para lograr cambios en los hábitos y estilo de vida en gente sana.

Dra. Marcela Botargues [ Unidad de Medicina Familiar del Hospital Italiano de Buenos Aires ]

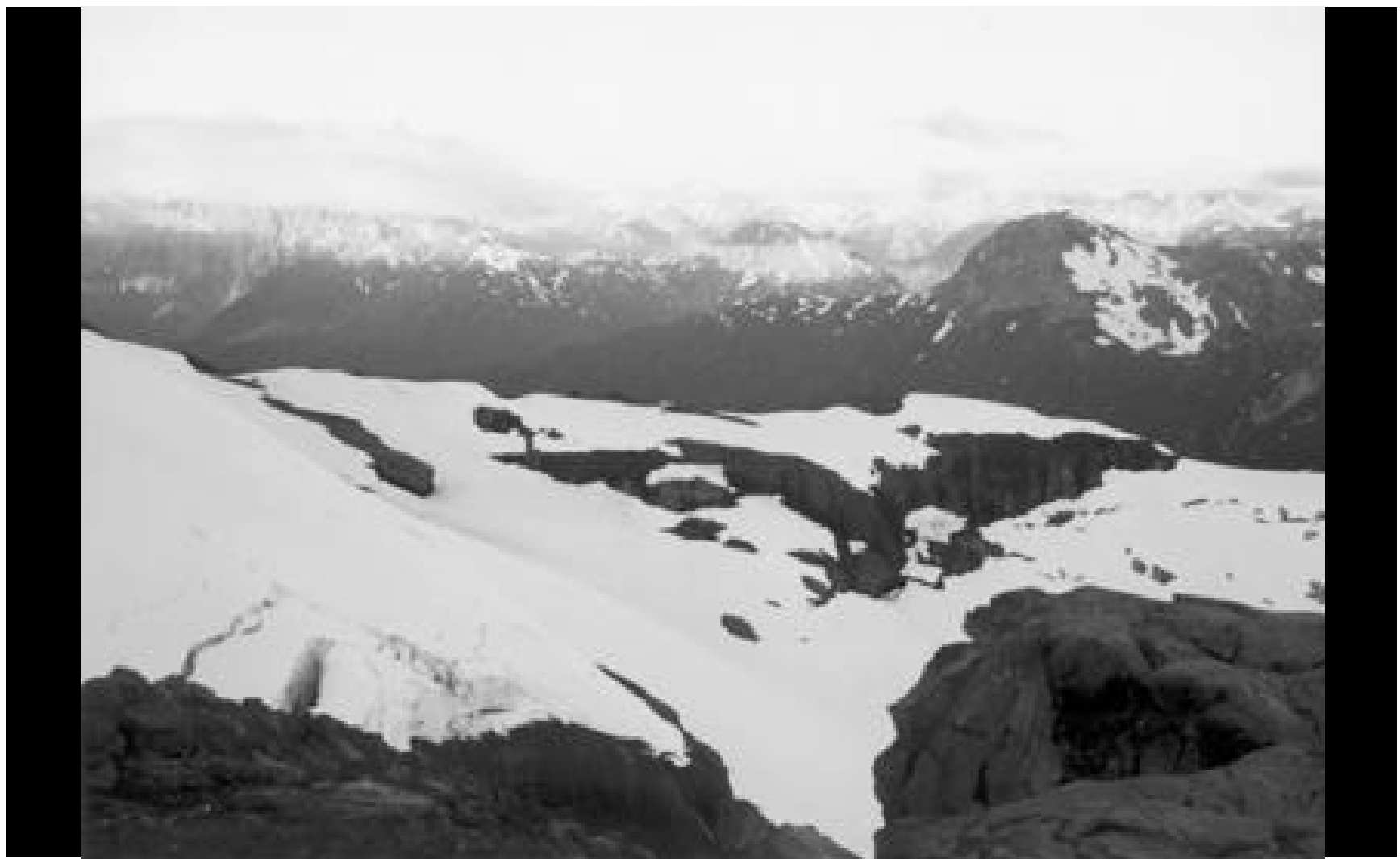

